\title{
On the Theory of Seismic and Seismoelectric Phenomena in a Moist Soil*
}

\author{
J. Frenkel ${ }^{\dagger}$
}

\section{Introduction}

A. G. Ivanov discovered in 1939 that the propagation of elastic waves in the surface layers of the soil is accompanied by an "electrification" of the latter or, more exactly, by the appearance of electric potential differences between points situated at different distances from the source of the waves. ${ }^{1}$ This phenomenon has been denoted by him as a "seismoelectric effect of the second kind" or " $E$-effect", in order to distinguish it from the effect of the first kind ( $J$ - effect), discovered previously by Blau and Statham, ${ }^{2}$ which consists in a variation of the strength of the current $J$, flowing through the soil in the presence of a constant potential difference between two electrodes under the influence of the elastic vibrations. The $J$-effect is due to a variation of the electric resistance of the soil under the influence of elastic vibrations and is, therefore, non-polar. According to A. Ivanov, the $E$-effect can be reduced to the electrofiltration effect in the disperse medium, which ordinary moist soil constitutes. The particles of the latter are immersed in water which plays the role of the dispersive medium. The boundary surface between these particles and the water is the seat of electric double layers, whose aqueous side has a diffuse structure. The presence of such layers explains the connection between the flow of water in the capillary spaces of the soil and the transfer of surface electrical charges which gives rise to an electrical field, in which case electrical currents are compensated by the volume conduction currents. According to the theory of Helmholtz and Smoluchovski ${ }^{2}$ the differences of hydrostatic pressure $\Delta p$ between two points of the soil must be connected with a difference of the electrical potential

$$
\Delta V=\frac{\varepsilon \zeta}{4 \pi \mu \sigma} \Delta p
$$

where $\zeta$ is the electrokinetic potential, i.e. the potential drop in the surface double layer, $\mu$ the viscosity of water and $\sigma$ its electrical conductivity. Since the propagation of longitudinal elastic

\footnotetext{
${ }^{*}$ Paper originally published in the Journal of Physics, Vol. III, No. 4, pp. 230-241, 1944. Republished with permission by the Schmidt Institute of Earth Physics, Russian Acaemy of Sciences, courtesy of Prof. Alecxander O. Gliko, Director. Paper prepared with typographical corrections by Alexander Cheng.

${ }^{\dagger}$ Physical Technical Institute and Institute of Theoretical Geophysics, Academy of the USSR.
} 
waves in the soil is accompanied by the variation of pressure in the direction of propagation, it must also be accompanied by a variation of the electric potential which constitutes the $E$-effect discovered by Ivanov. Its magnitude is of the order of $1 \mathrm{mV} / \mathrm{m}$ which is somewhat smaller than the theoretical value obtained from formula (1) for clay soils, and by a factor some tenths times smaller than the corresponding value for limestones.

According to Ivanov's experiments, the electrode situated closer to the explosion acquires at the initial instant of the transition of the elastic wave a negative potential with respect to the more distant electrode. This circumstance shows that the particles of the soil have a positive charge while the adherent water layers - a negative one, in accordance with the results of direct measurements of soil suspensions.

Although Ivanov's considerations are thus essentially correct, a quantitative theory of his "E-effect" can only be developed on the basis of the Helmholtz- Smoluchovski theory of filtration potentials by taking into account the following complications.

1. Equation (1) refers to the case of a steady flow of water through the pores; in the case of rapid vibrations account must be taken of the relaxation time, i.e. of the fact that the conduction current, due to the potential drop $\Delta V$ which compensates the surface current giving rise to this potential drop, is established within a finite lapse of time.

2. Equation (1) derived for the case of absolutely rigid filter with invariable pores, whereas the propagation of longitudinal elastic waves in the soil is connected with a periodical compression and expansion both of the particles, constituting it, and of the pores between them, as well as of the liquid filling these pores.

3. The electrical oscillations, due to the mechanical vibrations of the soil, in connection with the relative motion of its particles and of the dispersive medium and with the presence of charges of opposite signs must, in their turn, react on these mechanical vibrations and modify their propagation.

The second point is of special interest, apart from its connection with the filtration potentials. The modern theory of the motion of the water in the soil based on Darcy's law does not take into account the fact that the particles of the soil can be elastically compressed and extended, assuming that the external forces and the hydrostatic pressure act on the liquid filling these pores only. This simplifying assumption necessitates a correction even in the case of problems on the steady flow of soil water under the influence of given external forces. It becomes, however, wholly untenable in the case of such questions as the propagation of elastic vibrations in the soil. It should be noted in this connection that the velocity of sound in the soil is usually less than $1 \mathrm{~km} / \mathrm{sec}$, whereas in deeper layers of the earth, which are practically non-porous and dry it reaches $6-8 \mathrm{~km} / \mathrm{sec}$. As far as I am aware, this fact has not been subjected so far to a theoretical analysis. 
In the present paper we shall investigate this question and apply the results to a quantitative determination of the electric effect associated with the propagation of elastic vibrations.

\section{Statics of Dry Soil}

The soil, as a two-phase system, is characterized, from the point of view of its elastic properties, by a partial independence of its two components - the solid and the liquid one - from each other.

Let us assume, to begin with, that the liquid phase is wholly absent, the space formed by the pores remaining empty. The elastic properties of the soil can be described in this case from the macroscopic point of view by the ordinary equations of the elasticity theory of a solid amorphous body, namely:

$$
T_{i k}=\delta_{i k} L \theta+2 G u_{i k}
$$

where $T_{i k}(i, k=1,2,3)$ are the components of the elastic strain tensor, $\delta_{i k}=1$, for $i=k$ and $\delta_{i k}=0$ for $i \neq k$; the quantities

$$
u_{i k}=\frac{1}{2}\left(\frac{\partial u_{i}}{\partial x_{k}}+\frac{\partial u_{k}}{\partial x_{i}}\right)
$$

are the components of the strain tensor $\left(u_{1}, u_{2}, u_{3}\right.$ are components of the displacement of a particle with the coordinates $\left.x_{1}, x_{2}, x_{3}\right), \theta=\operatorname{div} \mathbf{u}=\Sigma \partial u_{i} / \partial x_{i}$ is the relative change of the effective volume of the dry soil. Finally, $L$ and $G$ are Lamé's coefficients, specifying the dry soil, i.e. a soil with empty pores. From equations (2) there follow the well known expressions for the components of the elastic force acting on an unit volume of the soil

$$
\Phi_{i}^{(1)}=\sum_{k} \frac{\partial T_{i k}}{\partial x_{k}}=L \frac{\partial \theta}{\partial x_{i}}+G \sum_{k} \frac{\partial^{2} u_{i}}{\partial x_{k}^{2}}+G \frac{\partial}{\partial x_{i}} \sum_{k} \frac{\partial u_{k}}{\partial x_{k}}
$$

or, in vector form

$$
\vec{\Phi}^{(1)}=(L+G) \nabla \theta+G \nabla^{2} \mathbf{u}
$$

In the macroscopic theory of the soil only such distances are considered, which are large compared with the linear dimensions of the solid particles or of the pores, and such elements of volume, which contain a very large number of these particles and pores. The presence and the degree of porosity is accounted for with the help of a certain coefficient $f$ equal to the ratio of the volume of pores $V_{2}$ to the total (macroscopical) volume, occupied by the soil $V_{1}+V_{2}=V$;

$V_{1}$ is here the volume actually filled by the solid particles constituting it. The actual density of these particles will be denoted by $\rho_{1}$ and the mean (macroscopical) density of the dry soil by $\gamma_{1}$. Referring the volumes $V_{1}$ and $V_{1}+V_{2}$ to unit mass, we have

$$
\rho_{1}=\frac{1}{V_{1}}, \quad \gamma_{1}=\frac{1}{V_{1}+V_{2}}
$$


and, consequently,

$$
\gamma_{1}=\rho_{1} \frac{V_{1}}{V_{1}+V_{2}}=\rho_{1}\left(1-\frac{V_{2}}{V_{1}+V_{2}}\right)
$$

i.e., according to the definition of $f$

$$
\gamma_{1}=\rho_{1}(1-f)
$$

The change of the specific volume of the soil $\Delta V$ consists of two parts: of the change of the volume of unit mass of the solid phase $\Delta V_{1}$ and of the change of the volume of the pores, connected with it, $\Delta V_{2}$. In the case of small strains which are dealt with in the elasticity theory, these quantities can be treated as proportional to each other, so that

$$
\Delta V_{1}=\alpha \Delta V_{2}
$$

where $\alpha$ is a proportionality coefficient, which along with the porosity $f$ specifies the mechanical properties of the dry soil. With the help of this parameter it is possible to express the variation of the degree of porosity of the soil due to its deformation. We have namely, according to the definition of $f$ :

$$
\Delta f=\frac{\Delta V_{2}}{\left(V_{1}+V_{2}\right)}-\frac{V_{2}\left(\Delta V_{1}+\Delta V_{2}\right)}{\left(V_{1}+V_{2}\right)^{2}}=\frac{V_{1} \Delta V_{2}-V_{2} \Delta V_{1}}{\left(V_{1}+V_{2}\right)^{2}}
$$

i.e.

$$
\frac{\Delta f}{f}=\frac{\frac{V_{1}}{V_{2}} \Delta V_{2}-\Delta V_{1}}{V_{1}+V_{2}}=\frac{\frac{V_{1}}{V_{2}}-\alpha}{V_{1}+V_{2}} \Delta V_{2}
$$

or since

$$
\begin{aligned}
\frac{V_{1}}{V_{2}} & =\frac{V_{1}+V_{2}}{V_{2}}-1=\frac{1}{f}-1=\frac{1-f}{f} \\
\Delta f & =\frac{1-f(1+\alpha)}{V_{1}+V_{2}} \Delta V_{2}
\end{aligned}
$$

On the other hand, according to the definition of the quantity $\theta$, we have

$$
\theta=\frac{\Delta V}{V}=\frac{\Delta V_{1}+\Delta V_{2}}{V_{1}+V_{2}}=\frac{(1+\alpha) \Delta V_{2}}{V_{1}+V_{2}}
$$

We thus get the following relation between $\Delta f$ and $\theta$ :

$$
\Delta f=\frac{1-f(1+\alpha)}{1+\alpha} \theta
$$

In the case of a large porosity the coefficient $\alpha$ must be small compared with unity, so that the compression or expansion of the soil is realized mainly at the cost of a compression or expansion of its pores. With the decrease of porosity $\alpha$ must increase; it is natural, however to assume 
that for all values of $f$ different from zero, the product $f(1+\alpha)$ must be smaller than unity, i.e. $\alpha<(1 / f)-1$.

The forces, acting on a solid body and characterized by the tensor $T_{i k}$, can be divided into a pressure $p_{1}=-(1 / 3)\left(T_{11}+T_{22}+T_{33}\right)$ and a shearing stress, specified by the tensor

$$
T_{i k}^{\prime}=T_{i k}-\delta_{i k} \frac{1}{3} \sum_{i} T_{i i}=T_{i k}+\delta_{i k} p_{1}
$$

In the absence of shearing stresses the deformation of the body is reduced to a simple expansion or compression, the pressure being defined by the formula:

$$
-p_{1}=L \theta+\frac{2}{3} G\left(u_{11}+u_{22}+u_{33}\right)=\left(L+\frac{2}{3} G\right) \theta
$$

which follows from equation (2) in connection with the definition of $\theta$. We thus get

$$
\theta=-\frac{1}{K} p_{1}
$$

where $K=L+(2 / 3) G$ is the compressibility modulus, and, consequently, according to (6)

$$
\Delta f=-\frac{1-f(1+\alpha)}{(1+\alpha) K} p_{1}
$$

Hence it is clear that, under the condition $\alpha<(1 / f)-1$, the compression of the dry soil must be accompanied by a decrease of its porosity, whereas in the case $\alpha>(1 / f)-1$ the contrary would be true. We shall also need in the sequel the relations

$$
u_{i k}=\delta_{i k} \sigma p_{1}+\tau T_{i k}
$$

which are obtained by solving equations (2) with respect to the quantities $u_{i k}$, the coefficients $\sigma$ and $\tau$ being defined by the formulae

$$
\sigma=\frac{L+\frac{4}{3} G}{2 G(L+2 G)}, \quad \tau=\frac{1}{2 G}
$$

The absence of shearing stresses is characterized by the equations $T_{11}=T_{22}=T_{33}=-p_{1}$ and $T_{12}=T_{23}=T_{31}=0$, formula (9) reducing in this case to (7).

\section{Statics of Moist Soil}

We have considered hitherto a completely dry soil. Let us now assume that all its pores are wholly filled with a liquid (water) which can flow in and out of them freely. What will be the influence of the liquid phase under such conditions on the macroscopic elastic properties of the soil? 
In order to remain in equilibrium the liquid phase must, in the absence of external forces, be subjected to the same hydrostatic pressure $p_{2}$ at all the points of the (multiply connected) space formed by the pores. This pressure must also be exerted on the solid skeleton of the soil. The resulting deformation of this skeleton must reduce to a variation of the volume of the solid phase in the same ratio as that of the liquid phase, according to the formula:

$$
\frac{\Delta V_{1}}{V_{1}}=\frac{\Delta V_{2}}{V_{2}}=-\frac{1}{K_{o}} p_{2}
$$

where $K_{o}$ is the true compressibility modulus of the solid phase. Hence it follows, firstly, that the whole (macroscopic) volume of the soil must be varied in the same ratio

$$
\frac{\Delta V}{V}=\theta=-\frac{1}{K_{o}} p_{2}
$$

and, secondly, that the degree of porosity must remain unaltered

$$
\Delta f=0
$$

A comparison of equations (7) and (10) shows that the hydrostatic pressure $p_{2}$ is equivalent, with respect to the compression of the soil produced by it, to a "solid pressure":

$$
p_{1}=\frac{K}{K_{o}} p_{2}
$$

which is smaller than $p_{2}$ since $K_{o}>K$.

Equation (10) must be completed by one more equation

$$
\frac{\Delta \rho_{2}}{\rho_{2}}=\frac{1}{K_{2}} p_{2}
$$

which connects the true density of the liquid with the hydrostatic pressure $\left(K_{2}\right.$ is the compressibility modulus of the liquid) and which represents the approximate form of the equation of state of the liquid at a constant temperature.

It must be kept in mind that the mass of the liquid $\rho_{2} V_{2}$ filling the pores in a certain part of the solid skeleton $V_{1}$ is, generally speaking, a variable quantity, in contradiction to the solid part $\rho_{1} V_{1}$ which, of course, remains constant. The pressure $p_{1}$ and $p_{2}$ are wholly different with respect to their origin and nature and completely independent of each other. The total variation of the macroscopic volume of the soil, due to their combined action, is obviously equal to the sum of the variations, due to each of them taken separately. We thus get, adding the expression (7) and (10)

$$
\theta=-\frac{1}{K} p_{1}-\frac{1}{K_{o}} p_{2}
$$

This formula refers to the particular case of the absence of shearing stresses in the solid skeleton of the soil. Inasmuch as such stresses are absent in a (resting) liquid the strain tensor of the soil 
is reduced, in the general case, to a sum of the expressions (9) and of the tensor $-\left(1 / 3 K_{o}\right) p_{2} \delta_{i k}$. Consequently, we have

$$
u_{i k}=\delta_{i k}\left(\sigma p_{1}-\frac{1}{3 K_{o}} p_{2}\right)+\tau T_{i k}
$$

These equations along with equations (12) determine the deformations of the solid and liquid phases as functions of the stresses. In order to derive the equations for the motion of the soil the stresses must be expressed as functions of the deformation (strain). Denoting $\Delta \rho_{2} / \rho_{2}$ by $\varphi$ ( $\varphi$ plays for the liquid phase the same role as $\theta$ for the solid one) and replacing $p_{2}$ according to (12), by $-K_{2} \varphi$ we can rewrite equations (14) in the form

$$
u_{i k}-\delta_{i k} \frac{K_{2}}{3 K_{o}} \varphi=\delta_{i k} \sigma p_{1}+\tau T_{i k}
$$

Comparing with them (9) we see that, with regard to the hydrostatic pressure, the stress tensor in the solid skeleton $T_{i k}$ must be expressed through the quantities $u_{i k}-\delta_{i k}\left(K_{2} / 3 K_{o}\right) \varphi$ in the same way, as it is expressed through $u_{i k}$ when $\varphi=0$. Noting that $\theta=\sum_{i} u_{i i}$ is replaced in this case by $\theta=\left(K_{2} / K_{o}\right) \varphi$, and modifying equations (2) in the way indicated, we obtain the following generalized expressions for $T_{i k}$ :

$$
T_{i k}=\delta_{i k}\left(L \theta-\frac{K_{2}}{K_{o}} \varphi\right)+2 G\left(u_{i k}-\delta_{i k} \frac{K_{2}}{3 K_{o}} \varphi\right)
$$

or, since $L+(2 / 3) G=K$,

$$
T_{i k}=\delta_{i k}\left(L \theta-\frac{K K_{2}}{K_{o}} \varphi\right)+2 G u_{i k}
$$

These expressions along with the equation of state of the liquid phase

$$
p_{2}=-K_{2} \varphi
$$

enable one to determine the volume elastic forces acting on the soil in that case when the quantities $T_{i k}$ and $p_{2}$ vary from one point to another. In the ordinary elasticity theory the components of the elastic force, referred to a unit volume of the body, are given by the formulae

$$
\Phi_{i}^{(1)}=\sum_{k} \frac{\partial T_{i k}}{\partial x_{k}}
$$

In our case this expression refers only to the solid phase, contained in a unit volume of the soil. In the presence of a hydrostatical pressure a unit volume of the soil is also acted upon by the force $-\nabla p_{2}$ which is distributed between the liquid and the solid phase in the ratio of the respective volumes, i.e. in the ratio $f /(1-f)$. The complete expression for $\Phi_{i}^{(1)}$ has thus the following form

$$
\Phi_{i}^{(1)}=\sum_{k} \frac{\partial T_{i k}}{\partial x_{k}}-(1-f) \frac{\partial p_{2}}{\partial x_{i}}
$$


whereas the force acting on the liquid phase in a unit volume of the soil is equal to

$$
\vec{\Phi}^{(2)}=-f \nabla p_{2}
$$

Substituting into these formulae the expressions (15) and (15a) we get

$$
\begin{aligned}
\Phi_{i}^{(1)} & =\frac{\partial}{\partial x_{i}}\left(L \theta-\frac{K K_{2}}{K_{o}} \varphi\right)+G\left(\sum_{k} \frac{\partial^{2} u_{i}}{\partial x_{k}^{2}}+\frac{\partial}{\partial x_{i}} \sum_{k} \frac{\partial u_{k}}{\partial x_{k}}\right)+(1-f) K_{2} \frac{\partial \varphi}{\partial x_{i}} \\
& =\frac{\partial}{\partial x_{i}}\left[(L+G) \theta-\frac{K K_{2}}{K_{o}} \varphi\right]+G \sum_{k} \frac{\partial^{2} u_{i}}{\partial x_{k}^{2}}+(1-f) K_{2} \frac{\partial \varphi}{\partial x_{i}}
\end{aligned}
$$

or in a vector form

$$
\vec{\Phi}^{(1)}=(L+G) \nabla \theta+G \nabla^{2} \mathbf{u}+K_{2}\left(1-f-\frac{K}{K_{o}}\right) \nabla \varphi
$$

and

$$
\vec{\Phi}^{(2)}=f K_{2} \nabla \varphi
$$

\section{The Equations of Motion of the Soil with Account of the Friction between the Solid and the Liquid Phases}

In modern agrophysics and hydrology the mean velocity of the flow of the liquid phase (water, oil, etc.), filing the pores between the particles of the soil, on the assumption of the absolute rigidity of the solid skeleton formed by these particles, is determined by Darcy's equation

$$
\mathbf{v}_{2}=\frac{k}{\mu}\left(-\nabla p_{2}+\mathbf{F}_{2}\right)
$$

where $\mathbf{F}_{2}$ denotes the external force acting on the liquid contained in a unit volume of the soil, $\mu$ the viscosity coefficient of this liquid and $k$ the filtration coefficient of the soil. The latter is proportional to the degree of its porosity $f$ and to the mean value of the cross section of the pores, i.e. to the square of their linear dimensions $\delta$

$$
k=\text { const } \cdot f \delta^{2}
$$

Equation (18) must be completed, just as in ordinary hydrodynamics, by the continuity equation

$$
\frac{\partial \gamma_{2}}{\partial t}+\operatorname{div}\left(\gamma_{2} \mathbf{v}_{2}\right)=0
$$

where $\gamma_{2}=f \rho_{2}$ is the mean density of the liquid in a macroscopically small region, containing, however, a large number of pores. Some authors replace $\gamma_{2}$ in the second term of the left-hand side by $\rho_{2}$ writing the continuity equation in the form

$$
f \frac{\partial \rho_{2}}{\partial t}+\operatorname{div}\left(\rho_{2} \mathbf{v}_{2}\right)=0
$$


It can easily be seen, however, that in this form it contradicts the law of conservation of the mass of the liquid, in connection with the definition of $\mathbf{v}_{2}$ as the mean macroscopic velocity of its flow. This circumstance is of no importance so long as the liquid is dealt with as incompressible or if the solid skeleton of the soil is treated as absolutely rigid (in the latter case the definition of $\mathbf{v}_{2}$ must be slightly altered). In the case of a deformable skeleton, however, the application of equation (20a) must lead to erroneous results.

Equation (18) refers to the case of a steady flow. In the case of a variable flow it is replaced by the following one:

$$
\gamma_{2} \frac{\partial \mathbf{v}_{2}}{\partial t}=-\nabla p_{2}+\mathbf{F}_{2}-\frac{\mu}{k} \mathbf{v}_{2}
$$

The quadratic term $\left(\mathbf{v}_{2} \cdot \nabla\right) \mathbf{v}_{2}$ can, of course, be neglected. This equation is, however, inexact, because of the absence of the factor $f$ at the gradient of the pressure $p_{2}$. Introducing this factor, we obtained the corrected equation for the motion of the liquid

$$
\gamma_{2} \frac{\partial \mathbf{v}_{2}}{\partial t}=-f \nabla p_{2}+\mathbf{F}_{2}-\frac{\mu}{\kappa} \mathbf{v}_{2}
$$

where the ordinary filtration coefficient $k$ is replaced by the coefficient

$$
\kappa=\frac{k}{f}=\mathrm{const} \cdot \delta^{2}
$$

which ensures Darcy's law in its usual statement (18) for the special case of a steady flow of the liquid.

Equation (21) is easily generalized to the case, when the deformability and the mobility of the solid skeleton becomes important, e.g. in the propagation of elastic vibrations. The absolute velocity of the liquid $\mathbf{v}_{2}$ must be replaced by relative velocity with respect to the solid phase $\mathbf{v}_{2}-\mathbf{v}_{1}$ where $\mathbf{v}_{1}=\partial \mathbf{u} / \partial t$ denotes the mean macroscopic velocity of the particles of the solid phase at the corresponding point. This relative velocity is connected with the friction force acting on the liquid in unit volume of the soil, by the relation

$$
\mathbf{F}_{21}=-\frac{\mu}{\kappa}\left(\mathbf{v}_{2}-\mathbf{v}_{1}\right)
$$

the solid phase being acted on the part of the liquid one by the opposite force $\mathbf{F}_{12}=-\mathbf{F}_{21}$ (referred likewise to a unit volume of the soil).

Replacing $\mathbf{v}_{2}$ in equation (21) by $\mathbf{v}_{2}-\mathbf{v}_{1}$ and $p_{2}$ by $-K_{2} \varphi$ we obtain finally

$$
\gamma_{2} \frac{\partial \mathbf{v}_{2}}{\partial t}=f K_{2} \nabla \varphi+\mathbf{F}_{2}-\frac{\mu}{\kappa}\left(\mathbf{v}_{2}-\mathbf{v}_{1}\right)
$$

In the case of the absence of external forces this equation can be rewritten in the from

$$
\rho_{2} \frac{\partial \mathbf{v}_{2}}{\partial t}=K_{2} \nabla \varphi-\frac{\mu}{\kappa f}\left(\mathbf{v}_{2}-\mathbf{v}_{1}\right)
$$


The equation of motion of the solid phase in the general case of a relative motion of the liquid can be written as follows

$$
\gamma_{1} \frac{\partial \mathbf{v}_{1}}{\partial t}=\vec{\Phi}^{(1)}+\mathbf{F}_{1}+\frac{\mu}{\kappa}\left(\mathbf{v}_{2}-\mathbf{v}_{1}\right)
$$

Since the velocity is treated in the elastic theory as a function of the time and of the initial coordinates of the corresponding particle, $\partial \mathbf{v}_{1} / \partial t$ is the exact value of the acceleration, in contradistinction to the quantity $\partial \mathbf{v}_{2} / \partial t$ referring to the liquid phase; the exact expression for the acceleration being in the latter case $\left(\partial \mathbf{v}_{2} / \partial t\right)+\left(\mathbf{v}_{2} \cdot \nabla\right) \mathbf{v}_{2}$. In practice, however, the motion of the liquid is so slow that this difference does not play any role, the quadratic term being negligibly small. It should be mentioned also that the effective density of the solid phase $\gamma_{1}=(1-f) \rho_{1}$ in the equation (23) refers to the unstrained state and must therefore be treated as a constant quantity.

Substituting expression (17) for $\vec{\Phi}^{(1)}$ in (23) we get, in the absence of external forces:

$$
\gamma_{1} \frac{\partial \mathbf{v}_{1}}{\partial t}=(L+G) \nabla \theta+G \nabla^{2} \mathbf{u}+K_{2}\left(1-f-\frac{K}{K_{o}}\right) \nabla \varphi+\frac{\mu}{\kappa}\left(\mathbf{v}_{2}-\mathbf{v}_{1}\right)
$$

The equations (22) or (22a) and (23a) contain the following quantities: $\mathbf{u}, \mathbf{v}_{2}, \rho_{2}, \varphi$ and $f$ $\left(\mathbf{v}_{1}=\partial \mathbf{u} / \partial t, \theta=\operatorname{div} \mathbf{u}, \gamma_{1}=\right.$ const $)$. The quantities $\rho_{2}$ and $\varphi$ are connected by the relation

$$
\rho_{2}=\rho_{o}(1-\varphi)
$$

where $\rho_{0}=\rho_{2}^{o}$ is the normal density of the liquid; its effective density is connected with the velocity $\mathbf{v}_{2}$ by the continuity equation (20); finally, the variation of the degree of porosity is connected with $\theta$ by the relation

$$
\Delta f=\frac{1-f(1+\alpha)}{(1+\alpha)}\left(\theta-\frac{K_{2}}{K_{o}} \varphi\right)
$$

This relation is obtained from (6) if $\theta$ is replaced by that part $\theta=-p_{1} / K$ which is due to the "solid" pressure and which, as has been shown in the derivation of equation (15), is equal to $\theta-\left(K_{2} / K_{o}\right) \varphi$.

We thus get for the determination of the five unknowns a system of five equations, so that our problem of the integration of the equations of motion of the soil is fully determined.

\section{Propagation of Longitudinal Vibrations in the Soil}

We shall limit ourselves in the sequel to the investigation of the question of the propagation of the small vibrations in the soil and shall, accordingly, linearize the equations of motion, i.e. replace the coefficients of the small quantities (such as $\mathbf{u}, \mathbf{v}_{1}, \mathbf{v}_{2}, \theta, \varphi$ and $\Delta f$ ) by their normal values, referring to the unstrained phase, just as is done in the ordinary hydrodynamics and 
elasticity theory. For the investigation of longitudinal vibrations we shall apply the operation div to the equations of motion. We shall make use of the following formula

$$
\operatorname{div} \mathbf{v}_{1}=\frac{\partial}{\partial t} \operatorname{div} \mathbf{u}=\frac{\partial \theta}{\partial t}
$$

and further of the equation

$$
\operatorname{div} \mathbf{v}_{2}=-\frac{1}{\gamma_{2}} \frac{\partial \gamma_{2}}{\partial t}=-\frac{1}{f} \frac{\partial f}{\partial t}-\frac{1}{\rho_{2}} \frac{\partial \rho_{2}}{\partial t}=-\frac{1}{f} \frac{\partial \Delta f}{\partial t}+\frac{\partial \varphi}{\partial t}
$$

following from equation (20) in a linearized form and which in connection with (11) and (24a) can be written in the form

$$
\operatorname{div} \mathbf{v}_{2}=-(\beta-1) \frac{\partial \theta}{\partial t}+\beta^{\prime} \frac{\partial \varphi}{\partial t}
$$

where for the sake of brevity we have put

$$
\beta=\frac{1}{f(1+\alpha)}, \quad \beta^{\prime}=1+(\beta-1) \frac{K_{2}}{K_{o}}
$$

Applying the operation div to both sides of the linearized equation (23a) we get with the help of the preceding formulae

$$
\frac{\partial^{2} \theta}{\partial t^{2}}=\frac{E}{\gamma_{1}} \nabla^{2} \theta+\frac{K_{2}}{\gamma_{1}}\left(1-f-\frac{K}{K_{o}}\right) \nabla^{2} \varphi+\frac{\mu}{\kappa \gamma_{1}}\left(\beta^{\prime} \frac{\partial \varphi}{\partial t}-\beta \frac{\partial \theta}{\partial t}\right)
$$

where $E=L+2 G$ is Young's modulus for dry soil.

In a similar way equation (22a) gives

$$
\frac{\partial^{2} \varphi}{\partial t^{2}}-\frac{\beta-1}{\beta^{\prime}} \frac{\partial^{2} \theta}{\partial t^{2}}=\frac{K_{2}}{\beta^{\prime} \rho_{2}} \nabla^{2} \varphi-\frac{\mu}{\kappa \gamma_{2}}\left(\frac{\partial \varphi}{\partial t}-\frac{\beta}{\beta^{\prime}} \frac{\partial \theta}{\partial t}\right)
$$

Equations (27) and (27a) contain in principle the solution of the question of the propagation of longitudinal vibrations in a moist soil.

Before turning to their integration we shall note an important special or rather limiting case corresponding to an extremely large value of the parameter $\mu / \kappa \gamma_{2}$, i.e. to an extreme smallness of the pores. Dividing equations (27) and (27a) by this parameter and noting that the quantities $\theta$ and $\varphi$ must have finite values, we obtain in this case the following relation between them

$$
\varphi=\frac{\beta}{\beta^{\prime}} \theta
$$

This relation means obviously that the two velocities $\mathbf{v}_{1}$ and $\mathbf{v}_{2}$ are identical, there being no relative motion of the liquid with respect to the solid.

Under the conditions (28) equation (27) is reduced to

$$
\frac{\partial^{2} \theta}{\partial t^{2}}=\left(\frac{E}{\gamma_{1}}+\varepsilon \frac{\beta}{\beta^{\prime}}\right) \nabla^{2} \theta
$$


where

$$
\varepsilon=\frac{K_{2}}{\gamma_{1}}\left(1-f-\frac{K}{K_{o}}\right)
$$

while equation $(27 \mathrm{a})$ is reduced to

$$
\frac{\partial^{2} \varphi}{\partial t^{2}}=\frac{\beta}{\beta^{\prime}} \frac{K_{2}}{\rho_{2}} \nabla^{2} \varphi
$$

The latter equation obviously contradicts equation (29), since the functions $\theta$ and $\varphi$ must be connected by the relation (28) - unless the velocity of the propagation of waves, described by equation (29), i.e. $\sqrt{\left(E / \gamma_{1}\right)+\left(\varepsilon \beta / \beta^{\prime}\right)}$, coincides accidentally with the velocity of the waves, described by equations $(30) \sqrt{\left(K_{2} / \rho_{2}\right)\left(\beta / \beta^{\prime}\right)}$.

Leaving aside this case we see that, when the parameter $\mu / \kappa$ (or $\left.\mu / \kappa \gamma_{2}\right)$ tends to infinity, the difference of the velocities $\mathbf{v}_{2}-\mathbf{v}_{1}$ or of their divergences tends to zero in such a way that its product with this parameter remains finite.

Keeping this in mind we shall represent this difference in the from of a series of powers of the small parameter $\kappa / \mu=\zeta$, i.e. we shall put

$$
\varphi=\frac{\beta}{\beta^{\prime}} \theta+\zeta \psi_{1}+\zeta^{2} \psi_{2}+\zeta^{3} \psi_{3}+\ldots
$$

where $\psi_{1}, \psi_{2}, \ldots$ are certain unknown functions (with finite values).

Before substituting this expression in equations (27) and (27a) it must de noted that in solving these equations by the method of successive approximations the function $\theta$ must also be expanded in a series of powers of $\zeta$, i.e.

$$
\theta=\theta_{0}+\zeta \theta_{1}+\zeta^{2} \theta_{2}+\ldots
$$

and, consequently,

$$
\varphi=\frac{\beta}{\beta^{\prime}} \theta_{0}+\zeta\left(\frac{\beta}{\beta^{\prime}} \theta_{1}+\psi_{1}\right)+\zeta^{2}\left(\frac{\beta}{\beta^{\prime}} \theta_{2}+\psi_{2}\right)+\ldots
$$

Substituting this expression in equation (27), (27a) and equating in each of them the coefficients of the same powers of $\zeta$ on both sides, we obtain a system of equations for the determination of functions $\theta_{k}$ and $\psi_{k}$. In the zero approximation (i.e., confining ourselves to terms not containing the parameter $\zeta$ ) we have, making use of the notations (29a)

$$
\begin{aligned}
& \frac{\partial^{2} \theta_{0}}{\partial t^{2}}=\left(\frac{E}{\gamma_{1}}+\varepsilon \frac{\beta}{\beta^{\prime}}\right) \nabla^{2} \theta_{0}+\frac{\beta^{\prime}}{\gamma_{1}} \frac{\partial \psi_{1}}{\partial t} \\
& \frac{\partial^{2} \theta_{0}}{\partial t^{2}}=\frac{K_{2}}{\rho_{2}} \frac{\beta}{\beta^{\prime}} \nabla^{2} \theta_{0}-\frac{\beta^{\prime}}{\gamma_{2}} \frac{\partial \psi_{1}}{\partial t}
\end{aligned}
$$


Multiplying the first of these equations by $\gamma_{1}$ and the second by $\gamma_{2}$ and adding them, we obtain the following equation for $\theta_{0}$ :

$$
\left(\gamma_{1}+\gamma_{2}\right) \frac{\partial^{2} \theta_{0}}{\partial t^{2}}=\left(E+\varepsilon \frac{\beta}{\beta^{\prime}} \gamma_{1}+K_{2} f \frac{\beta}{\beta^{\prime}}\right) \nabla^{2} \theta_{0}
$$

i.e., according to the definition of $\varepsilon$,

$$
\left(\gamma_{1}+\gamma_{2}\right) \frac{\partial^{2} \theta_{0}}{\partial t^{2}}=\left[E+\frac{\beta}{\beta^{\prime}} K_{2}\left(1-\frac{K}{K_{o}}\right)\right] \nabla^{2} \theta_{0}
$$

This equation describes waves, which are propagated without damping with the velocity

$$
w_{o}=\sqrt{\frac{E+\frac{\beta}{\beta^{\prime}} K_{2}\left(1-\frac{K}{K_{o}}\right)}{\gamma_{1}+\gamma_{2}}}
$$

In order to obtain the next approximation, we must, substituting expressions (31a) and (31b) in equations (27) and (27a), preserve and equate one another the first order terms. As a result we obtain the system of equations

$$
\begin{gathered}
\frac{\partial^{2} \theta_{1}}{\partial t^{2}}=\left(\frac{E}{\gamma_{1}}+\varepsilon \frac{\beta}{\beta^{\prime}}\right) \nabla^{2} \theta_{1}+\varepsilon \nabla^{2} \psi_{1}+\frac{\beta^{\prime}}{\gamma_{1}} \frac{\partial \psi_{2}}{\partial t} \\
\frac{\partial^{2} \theta_{1}}{\partial t^{2}}+\beta^{\prime} \frac{\partial^{2} \psi_{1}}{\partial t^{2}}=\frac{K_{2}}{\rho_{2}} \nabla^{2}\left(\frac{\beta}{\beta^{\prime}} \theta_{1}+\psi_{1}\right)-\frac{\beta^{\prime}}{\gamma_{2}} \frac{\partial \psi_{2}}{\partial t}
\end{gathered}
$$

Multiplying the former by $\gamma_{1}$ and the latter by $\gamma_{2}$ and adding, we obtain the following relation between $\theta_{1}$ and $\psi_{1}$ (or $\theta_{1}$ and $\varphi_{1}$ ):

$$
\left(\gamma_{1}+\gamma_{2}\right) \frac{\partial^{2} \theta_{1}}{\partial t^{2}}-\left[E+\frac{\beta}{\beta^{\prime}}\left(\varepsilon \gamma_{1}+\frac{K_{2}}{\rho_{2}} \gamma_{2}\right)\right] \nabla^{2} \theta_{1}=-\beta^{\prime} \gamma_{2} \frac{\partial^{2} \psi_{1}}{\partial t^{2}}+\left(\varepsilon \gamma_{1}+\frac{K_{2}}{\rho_{2}} \gamma_{2}\right) \nabla^{2} \psi_{1}
$$

or, according to (33a)

$$
\frac{\partial^{2} \theta_{1}}{\partial t^{2}}-w_{o}^{2} \nabla^{2} \theta_{1}=-\frac{\beta^{\prime} \gamma_{2}}{\gamma_{1}+\gamma_{2}} \frac{\partial^{2} \psi_{1}}{\partial t^{2}}+\frac{\varepsilon \gamma_{1}+K_{2} f}{\gamma_{1}+\gamma_{2}} \nabla^{2} \psi_{1}
$$

i.e., in virtue of the definition of $\varepsilon$ [formula (29a)]:

$$
\frac{\partial^{2} \theta_{1}}{\partial t^{2}}-w_{o}^{2} \nabla^{2} \theta_{1}=-\frac{\beta^{\prime} \gamma_{2}}{\gamma_{1}+\gamma_{2}} \frac{\partial^{2} \psi_{1}}{\partial t^{2}}+\frac{K_{2}\left(1-\frac{K}{K_{o}}\right)}{\gamma_{1}+\gamma_{2}} \nabla^{2} \psi_{1}
$$

From a comparison of this equation with equation (33), there follows among other things that its right-hand side must be orthogonal to the function $\theta_{0}$. For the determination of $\theta_{1}$ the 
function $\psi$ in (35) must be replaced by its expression through $\theta_{0}$ according to one of equations (32). We thus get

$$
\begin{aligned}
\frac{\partial}{\partial t}\left(\frac{\partial^{2} \theta_{1}}{\partial t^{2}}-w_{o}^{2} \nabla^{2} \theta_{1}\right)= & -\frac{\gamma_{1} \gamma_{2}}{\gamma_{1}+\gamma_{2}} \frac{\partial^{2}}{\partial t^{2}}\left[\frac{\partial^{2} \theta_{0}}{\partial t^{2}}-\left(\frac{E}{\gamma_{1}}+\varepsilon \frac{\beta}{\beta^{\prime}}\right) \nabla^{2} \theta_{0}\right] \\
& +\frac{\gamma_{1} K_{2}\left(1-\frac{K}{K_{o}}\right)}{\beta^{\prime}\left(\gamma_{1}+\gamma_{2}\right)} \nabla^{2}\left[\frac{\partial^{2} \theta_{0}}{\partial t^{2}}-\left(\frac{E}{\gamma_{1}}+\varepsilon \frac{\beta}{\beta^{\prime}}\right) \nabla^{2} \theta_{0}\right]
\end{aligned}
$$

This process can easily be continued in order to obtain approximations of a higher order.

\section{Special Case of Longitudinal Plane Sine Waves}

We shall not dwell on a more detailed consideration of this question in its general form but shall discuss the simplest special case of longitudinal harmonic vibrations propagated in the from of plane sine waves (with a small damping).

Let us assume accordingly that the dependence of the quantities $\theta$ and $\varphi$ on the time and the coordinate $x$ is expressed by the factor

$$
e^{i(\omega t-q x)}
$$

where $\omega / 2 \pi=\nu$ is the frequency of the vibrations and $q / 2 \pi$ the complex wave number (equal in the absence of damping to the reciprocal of the wave length $\lambda$ ). The ratio $\omega / q$ is equal to the velocity (in general complex) of the propagation of the waves.

Under such conditions the differential equations (27) and (27a) are reduced to a system of two linear algebraic equations for the amplitudes $\theta$ and $\varphi$ :

$$
\begin{aligned}
\left(\frac{E}{\gamma_{1}} \xi-1+\frac{i \mu \beta}{\kappa \gamma_{1} \omega}\right) \theta+\left(\varepsilon \xi-\frac{i \mu \beta^{\prime}}{\kappa \gamma_{1} \omega}\right) \varphi & =0 \\
\left(\frac{\beta-1}{\beta^{\prime}}-\frac{i \mu \beta}{\kappa \gamma_{2} \beta^{\prime} \omega}\right) \theta+\left(\frac{K_{2}}{\beta^{\prime} \rho_{2}} \xi-1+\frac{i \mu}{\kappa \gamma_{2} \omega}\right) \varphi & =0
\end{aligned}
$$

where $\xi=q^{2} / \omega^{2}=1 / w^{2}$. This quantity, i.e. the propagation velocity of the wave, is determined by the quadratic equation

$$
\left(\frac{E}{\gamma_{1}} \xi-1+\frac{i \mu \beta}{\kappa \gamma_{1} \omega}\right)\left(\frac{K_{2}}{\beta^{\prime} \rho_{2}} \xi-1+\frac{i \mu}{\kappa \gamma_{2} \omega}\right)-\left(\varepsilon \xi-\frac{i \mu \beta^{\prime}}{\kappa \gamma_{1} \omega}\right)\left(\frac{\beta-1}{\beta^{\prime}}-\frac{i \mu \beta}{\kappa \gamma_{2} \beta^{\prime} \omega}\right)
$$

which represents the compatibility condition of equation (36). By elementary simplifications this equation is reduced to the form

$$
\begin{gathered}
\frac{E K_{2}}{\beta^{\prime} \gamma_{1} \rho_{2}} \xi^{2}-\left\{\frac{E}{\gamma_{1}}+\frac{K_{2}}{\rho_{2} \beta^{\prime}}+\frac{\beta-1}{\beta^{\prime}} \varepsilon-\frac{i \mu}{\kappa \omega} \frac{1}{\gamma_{1} \gamma_{2}}\left[E+\frac{\beta}{\beta^{\prime}} K_{2}\left(1-\frac{K}{K_{o}}\right)\right]\right\} \xi \\
+1-\frac{i \mu}{\kappa \omega}\left(\frac{1}{\gamma_{1}}+\frac{1}{\gamma_{2}}\right)=0
\end{gathered}
$$


We shall not write down the expression for its roots and shall only remark that for large values of the parameters $\zeta=\mu / \kappa$ one of them corresponds to waves with a very small damping, and the other - to waves with a very large damping. The waves of the second kind are thus really non-existent. For an approximate determination of the value $\xi$ corresponding to the waves of the first kind, let us represent $\xi$ in the from of a series of powers of the small parameter $i \omega \kappa / \mu=i \eta$ :

$$
\xi=\xi_{0}+i \eta \xi_{1}+\ldots
$$

Substituting this series into the left-hand side of equation (37) and equating to zero the coefficients of the various powers of $\eta$ starting with $\eta^{-1}$ we get

$$
\begin{gathered}
\frac{1}{\gamma_{1} \gamma_{2}}\left[E+\frac{\beta}{\beta^{\prime}} K_{2}\left(1-\frac{K}{K_{o}}\right)\right] \xi_{0}-\left(\frac{1}{\gamma_{1}}+\frac{1}{\gamma_{2}}\right)=0 \\
\frac{E K_{2}}{\beta^{\prime} \gamma_{1} \rho_{2}} \xi_{0}^{2}-\left(\frac{E}{\gamma_{1}}+\frac{K_{2}}{\rho_{2} \beta^{\prime}}+\frac{\beta-1}{\beta^{\prime}} \varepsilon\right) \xi_{0}-\frac{1}{\gamma_{1} \gamma_{2}}\left[E+\frac{\beta}{\beta^{\prime}} K_{2}\left(1-\frac{K}{K_{o}}\right)\right] \xi_{1}+1=0 \\
\frac{2 E K_{2}}{\beta^{\prime} \gamma_{1} \rho_{2}} \xi_{0} \xi_{1}-\left(\frac{E}{\gamma_{1}}+\frac{K_{2}}{\rho_{2} \beta^{\prime}}+\frac{\beta-1}{\beta^{\prime}} \varepsilon\right) \xi_{1}-\frac{1}{\gamma_{1} \gamma_{2}}\left[E+\frac{\beta}{\beta^{\prime}} K_{2}\left(1-\frac{K}{K_{o}}\right)\right] \xi_{2}=0
\end{gathered}
$$

and so on.

The first of these equations leads to expression (33) found before for the velocity of propagation of the waves $\left(\xi_{0}=1 / w_{0}^{2}\right)$.

Substituting it into the second equation we obtain the following expression for the first-order correction:

$$
i \eta \xi_{1}=i \eta \frac{\frac{E K_{2}}{\beta^{\prime} \gamma_{1} \rho_{2}} \xi_{0}^{3}-\left(\frac{E}{\gamma_{1}}+\frac{K_{2}}{\rho_{2} \beta^{\prime}}+\frac{\beta-1}{\beta^{\prime}} \varepsilon\right) \xi_{0}^{2}+\xi_{0}}{\left(\frac{1}{\gamma_{1}}+\frac{1}{\gamma_{2}}\right)}
$$

With an accuracy of the first order with respect to $\eta$ the complex velocity of the propagation of the waves $w$ is determined by the formulae

$$
\frac{1}{w}=\sqrt{\xi}=\xi_{0}^{1 / 2}\left(1+i \eta \frac{\xi_{1}}{\xi_{0}}\right)^{1 / 2} \approx \xi_{0}^{1 / 2}+i \eta \frac{\xi_{1}}{\xi_{0}^{1 / 2}}
$$

i.e.

$$
\frac{1}{w}=\frac{1}{w_{0}}+i \frac{\kappa}{\mu} \omega w_{0} \xi_{1}
$$

Inserting this expression in the exponent of the factor $\exp [i(\omega t-q x)]=\exp \left[i \omega\left(t-\frac{x}{w}\right)\right]$ and writing the latter in the form $\exp \left[i \omega\left(t-\frac{x}{w_{0}}\right)-\frac{1}{2} \delta x\right]$, where $\delta$ is the damping coefficient of the waves per unit length, we obtain for this coefficient the following expression

$$
\delta=\frac{\kappa \omega^{2}}{\mu w_{0}^{3}} \frac{\left(\frac{E}{\gamma_{1}}+\frac{K_{2}}{\beta^{\prime} \rho_{2}}+\frac{\beta-1}{\beta^{\prime}} \varepsilon\right)-w_{0}^{2}-\frac{E K_{2}}{\beta^{\prime} \gamma_{1} \rho_{2}} w_{0}^{-2}}{\gamma_{1}+\gamma_{2}}
$$


The damping coefficient thus proves to be proportional to the square of the frequency of the vibrations, just as in the case of the propagation of longitudinal waves in an ordinary viscous liquid.

Let us consider in conclusion the limiting case of the propagation of waves in a medium with a vanishing porosity. In this case $f \rightarrow 0$, i.e. consequently, $\gamma_{1}=\rho_{1}, \gamma_{2}=0$; further $\kappa \rightarrow 0, \beta=1 / f(1+\alpha)=1$ [this follows from equation (24a)]; $\beta^{\prime}=1$ and $K=K_{o}$. Under such

conditions $\delta$ vanishes and $w_{0}$ is reduced to $\sqrt{E / \rho_{1}}$, i.e. to the usual expression for the velocity of propagation of longitudinal waves in an isotropic elastic solid body.

Equation (33a) enables one to determine the variation of this velocity with an increase of the number and size of the pores, supposed to be filled with the liquid. An essential role is played here by the decrease of the elasticity modulus $K$ of the solid skeleton. We shall not dwell here on this question, for it requires a special investigation, which lies beyond the scope of the present paper.

\section{Propagation of Transversal Waves in a Moist Soil}

The equations which determine the laws of propagation of transversal vibrations in a moist soil can be obtained from the fundamental equations of motion (22) and (23a) by applying to them the operation rot. Introducing the notations $\frac{1}{2} \operatorname{rot} \mathbf{v}_{1}=\vec{\Omega}_{1}, \frac{1}{2} \operatorname{rot} \mathbf{v}_{2}=\vec{\Omega}_{2}$ (angular velocities of the elements of the solid skeleton and of the liquid phase) we get:

$$
\begin{aligned}
\gamma_{1} \frac{\partial^{2} \vec{\Omega}_{1}}{\partial t^{2}} & =G \nabla^{2} \vec{\Omega}_{1}+\frac{\mu}{\kappa} \frac{\partial}{\partial t}\left(\vec{\Omega}_{2}-\vec{\Omega}_{1}\right) \\
\gamma_{2} \frac{\partial \vec{\Omega}_{2}}{\partial t} & =-\frac{\mu}{\kappa} \frac{\partial}{\partial t}\left(\vec{\Omega}_{2}-\vec{\Omega}_{1}\right)
\end{aligned}
$$

In the case of plane sine waves propagated in the direction of the $x$ axis these differential equations reduce to the linear equations:

$$
\begin{aligned}
\left(G \xi-\gamma_{1}\right) \vec{\Omega}_{1} & =\frac{i \mu}{\kappa \omega}\left(\vec{\Omega}_{2}-\vec{\Omega}_{1}\right) \\
\gamma_{2} \vec{\Omega}_{2} & =\frac{i \mu}{\kappa \omega}\left(\vec{\Omega}_{2}-\vec{\Omega}_{1}\right)
\end{aligned}
$$

where $\xi=q^{2} / \omega^{2}$, while $q$ and $\omega$ have the same meaning as before. Eliminating from them $\vec{\Omega}_{1}$ and $\vec{\Omega}_{2}$ we obtain the following equation for $\xi$ :

$$
1=\frac{i}{\eta}\left(\frac{1}{\gamma_{2}}-\frac{1}{G \xi-\gamma_{1}}\right)
$$

whence it follows

$$
G \xi=\gamma_{1}+\frac{1}{\frac{1}{\gamma_{2}}+i \eta}
$$


or, in the first approximation (with respect to $\eta$ ):

$$
\xi=\frac{\gamma_{1}+\gamma_{2}}{G}-i \frac{\gamma_{2}^{2}}{G} \eta
$$

This formula shows that the transversal waves are propagated in a moist soil with the velocity

$$
w_{o \perp}=\sqrt{\frac{G}{\gamma_{1}+\gamma_{2}}}
$$

and with the damping coefficient

$$
\delta=\frac{\kappa}{\mu} \frac{\gamma_{2}^{2}}{\gamma_{1}+\gamma_{2}} \frac{\omega^{2}}{w_{o \perp}}
$$

which, just as in the case of longitudinal waves, is proportional to the square of the vibration frequency.

\section{Seismoelectric Effect}

The electrokinetic effect connected with the propagation of longitudinal waves in a moist soil can be calculated with the help of the formula

$$
E=-\frac{\varepsilon \zeta}{4 \pi \mu \sigma} \frac{\partial p}{\partial x}
$$

for the strength of the longitudinal electric field in the case of a steady flow of the liquid through the pores of the fixed rigid skeleton, this formula being obtained by differential equation (1) with respect to $x$.

In the case of a Poiseuille flow of the liquid through a capillary tube with a radius $r$, the average velocity of the flow $\bar{v}$ is connected with the pressure gradient by the relation

$$
\bar{v}=-\frac{r^{2}}{8 \mu} \frac{\partial p}{\partial x}
$$

Substituting the resulting expression for $-\partial p / \partial x$ into (44) we get

$$
E=\frac{2 \varepsilon \zeta}{\pi \sigma r^{2}} \bar{v}
$$

If the capillary is moved uniformly in the direction of its axis with a velocity $v_{1}$ this formula remains valid, if $v$ represents the relative velocity of the liquid, i.e. the difference

$$
\bar{v}=v_{2}-v_{1}
$$

We shall assume that this formula can be applied to the soil by a suitable choice of the effective radius of the pores $r$. Under such conditions the calculation of the electric field $E$ is reduced to the determination of the difference $v_{2}-v_{1}$. 
In the case of longitudinal waves this difference can be expressed through $\theta$ with the help of the relations (25) and (26), which assume the form

$$
i q v_{1}=i \omega \theta, \quad i q v_{2}=-i \omega(\beta-1) \theta+i \omega \beta^{\prime} \varphi
$$

We thus get

$$
v_{2}-v_{1}=\frac{\omega}{q}\left(\beta^{\prime} \varphi-\beta \theta\right)=w_{o}\left(\beta^{\prime} \varphi-\beta \theta\right)
$$

or, according to (31),

$$
v_{2}-v_{1}=w_{o} \beta^{\prime} \zeta \psi_{1}=\frac{\kappa w_{o}}{\mu} \beta^{\prime} \psi_{1}
$$

The function $\psi$ can be expressed through $\theta_{0}$ (the value of $\theta$ in the zero approximation) with the help of one of expressions (32), which in the case under consideration is reduced to

$$
-i \frac{\omega \beta^{\prime}}{\gamma_{2}} \psi_{1}=\left(\frac{K_{2}}{\rho_{2}} \frac{\beta}{\beta^{\prime}} q^{2}-\omega^{2}\right) \theta_{0}
$$

We thus get, in the first approximation

$$
\psi_{1}=i \omega \frac{\gamma_{2}}{\beta^{\prime}}\left(\frac{K_{2}}{\rho_{2}} \frac{\beta}{\beta^{\prime}} \xi_{0}-1\right) \theta_{0}
$$

whence

$$
v_{2}-v_{1}=\frac{i \omega \kappa}{\mu} \gamma_{2} w_{o}\left(\frac{K_{2}}{\rho_{2}} \frac{\beta}{\beta^{\prime}} \frac{1}{w_{o}^{2}}-1\right) \theta_{0}
$$

Replacing here $\theta=\partial u / \partial x$ by $-i q u$, where $u$ is the displacement of the particles of the soil, and substituting into (44), we obtain finally

$$
E=\frac{2 \varepsilon \zeta \kappa \omega^{2} f \rho_{2}}{\pi \sigma r^{2} \mu}\left(\frac{K_{2}}{\rho_{2}} \frac{\beta}{\beta^{\prime} w_{o}^{2}}-1\right) u
$$

Hence it is seen that, for a given value of the amplitude of the displacement $u$, the electric field is proportional to the square of the vibration frequency (just as the damping coefficient is). It follows further from (46) that the field $E$ is proportional to the degree of porosity $f$ (if the dependence of the ratio $\beta / \beta^{\prime}$ on $f$ can be neglected) and is independent of the radius of the pores (since the filtration coefficient $\kappa$ is proportional to the square of this radius $r$ ).

In the derivation of the equation (46) it has been tacitly assumed that the time $\tau$, required for the establishment of the kinetic potential gradient, is sufficiently small compared with the vibration periods $2 \pi / \omega$ so that the value of $E$ at any instant of time is practically identical with that, which corresponds to the instantaneous value of the relative velocity.

This assumption is probably valid up to the ultrasonic frequencies of the order of $10^{3} \mathrm{kc}$ and certainly holds in the case of seismic vibrations, which have a much lower frequency. The relaxation effects in the electric double layers on the boundary between the solid and the liquid 
phase can thus play a certain role in the propagation only of ultrasonic vibrations in artificial colloidal media with a solid skeleton.

It should be noted that such media are constituted by gels. Of special interest, from the point of view of the above theory, are thyxotropic gels of lyophobic colloids. We hope to come back to this question in a special paper.

It should be mentioned in conclusion that solid metallic bodies can also be treated as twophase systems similar to the soil, in which the role of the solid skeleton is played by the crystal lattice, composed of the positive ions, and the role of the liquid phase - by the "electron liquid". Hence it follows that the propagation of longitudinal elastic vibrations in a metallic body must be accompanied by electrical effects similar to those which are observed in the propagation of seismic vibrations in the soil. This question requires a special investigation.

(Translated by S. Frenkel.)

\section{References}

[1] A.G. Ivanov, Bull. Aca. Sci. URSS, série géographique et géophysique, No. 5, 699, 1940.

[2] See for example, Smoluchovski's paper in Graetz "Elektrizität und Magnetismus," Vol. II. 